\title{
Development and Evaluation
}

of Supercritical Fluid

Chromatography/Mass

Spectrometry for Polar and

High-Molecular-Weight

Coal Components

Technical Progress Report

E. K. Chess

R. D. Smith

January 1986

Prepared for

the U.S. Department of Energy

Morgantown Energy Technology Center

under Contract DE-AC06-76RLO 1830

Pacific Northwest Laboratory

Operated for the U.S. Department of Energy

by Battelle Memorial Institute 


\section{DISCLAIMER}

This report was prepared as an account of work sponsored by an agency of the United States Government. Neither the United States Government nor any agency thereof, nor any of their employees, makes any warranty, express or implied, or assumes any legal liability or responsibility for the accuracy, completeness, or usefulness of any information, apparatus, product, or process disclosed, or represents that its use would not infringe privately owned rights. Reference herein to any specific commercial product, process, or service by trade name, trademark, manufacturer, or otherwise, does not necessarily constitute or imply its endorsement, recommendation, or favoring by the United States Government or any agency thereof. The views and opinions of authors expressed herein do not necessarily state or reflect those of the United States Government or any agency thereof.

\section{PACIFIC NORTHWEST LABORATORY \\ operated by \\ BATTELLE \\ for the \\ UNITED STATES DEPARTMENT OF ENERGY \\ under Contract DE-AC06-76RLO 1830}

\begin{tabular}{|c|c|}
\hline \multicolumn{2}{|c|}{ Printed in the United States of America } \\
\hline \multicolumn{2}{|c|}{$\begin{array}{l}\text { Available from } \\
\text { A }\end{array}$} \\
\hline \multirow{2}{*}{\multicolumn{2}{|c|}{$\begin{array}{l}\text { National Technical Information Service } \\
\text { United States Department of Commerce }\end{array}$}} \\
\hline & \\
\hline \multicolumn{2}{|c|}{$\begin{array}{c}5285 \text { Port Royal Road } \\
\text { Springfield, Virginia } 22161\end{array}$} \\
\hline \multirow{2}{*}{\multicolumn{2}{|c|}{$\begin{array}{l}\text { NTIS Price Codes } \\
\text { Microfiche A01 }\end{array}$}} \\
\hline & \\
\hline \multicolumn{2}{|c|}{ Printed Copy } \\
\hline & \\
\hline Pages & Codes \\
\hline $001-025$ & $\mathrm{~A} 02$ \\
\hline 026-050 & $\mathrm{A} 03$ \\
\hline 051.075 & A04 \\
\hline $076-100$ & A05 \\
\hline $101-125$ & A06 \\
\hline $126-150$ & $\mathrm{~A} 07$ \\
\hline 151.175 & $A 08$ \\
\hline $176-200$ & $\mathrm{~A} 09$ \\
\hline $201-225$ & A010 \\
\hline $226-250$ & A011 \\
\hline $251-275$ & A012 \\
\hline $276-300$ & A 013 \\
\hline
\end{tabular}


DEVELOPMENT AND EVALUATION

OF SUPERCRITICAL FLUID

CHROMATOGRAPHY/MASS

SPECTROMETRY FOR POLAR AND

HIGH-MOLECULAR-WEIGHT

COAL COMPONENTS

Technical Progress Report

E. K. Chess

R. D. Smith

January 1986

Prepared for

the U.S. Department of Energy

Morgantown Energy Technology Center

under Contract DE-AC06-76RLO 1830

Pacific Northwest Laboratory

Richland, Washington 99352 


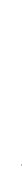


This Technical Progress Report reviews the technical progress made over the first 18 months of the program. Our goals include the design, development, and evaluation of a combined capillary column supercritical fluid chromatograph/high-performance mass spectrometer capable of analyzing high-molecular-weight polar materials and evaluating the system's potential for application in coal conversion process monitoring. The program includes not only the development and evaluation of the required instrumentation, but the development of polar fluids and compatible chromatographic stationary phases needed for efficient separation and analysis of polar and high-molecular-weight compounds. A new chromatograph/mass spectrometer interface and new mass spectrometer ion source have been designed, constructed, and evaluated using lowpolarity supercritical fluids such as pentane. Results from the evaluations have been used to modify the instrumentation to improve performance. The design and fabrication of capillary flow restrictors from fused silica tubing has been explored. Research has also been conducted toward advancing the technology of fabricating high-performance chromatographic columns suitable for use with polar supercritical fluids. Results to date support our initial belief that high-resolution supercritical fluid chromatography (SFC)/high-performance mass spectrometry (MS) will provide a significantly enhanced analytical capability for broad classes of previously intractable fuel components. 


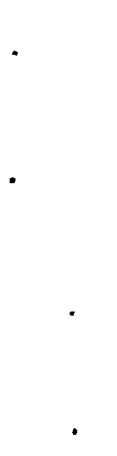




\section{CONTENTS}

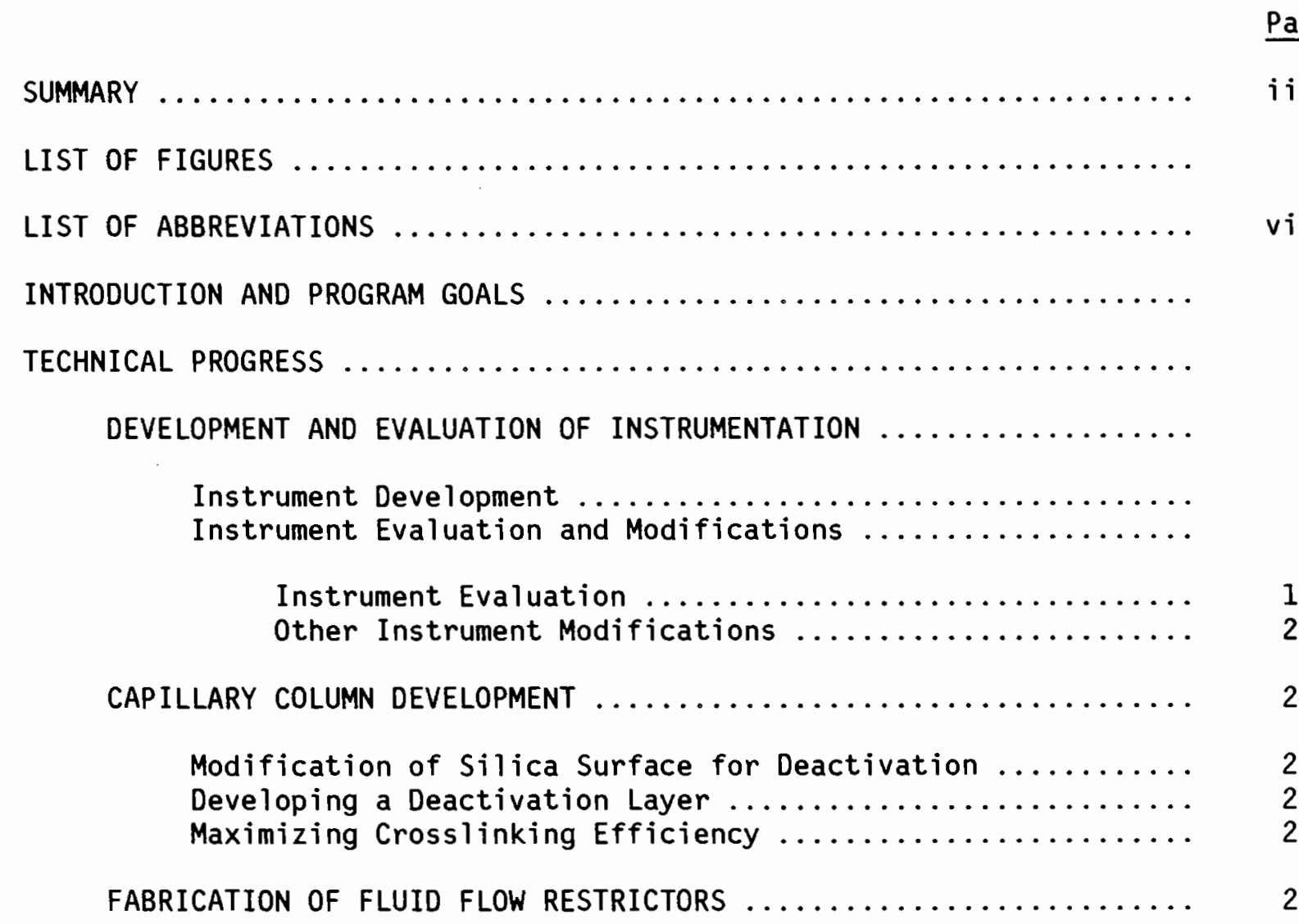

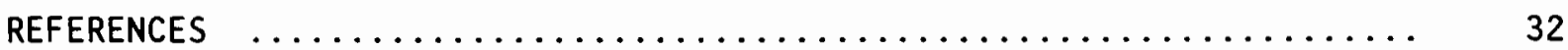

\section{LIST OF FIGURES}

1. Schematic Illustration of the Direct Fluid Injection Process .... 3

2. Schematic Illustration of the Capillary Column Supercritical Fluid Chromatograph/High-Resolution Mass Spectrometer System ....

3. Schematic Illustration of the Direct Fluid Injection/Supercritical Fluid Chromatography Oven, Interface Probe, and Ion Source..

4. Schematic Diagram of Modified Interface Probe $\ldots \ldots \ldots \ldots \ldots \ldots$

5. Photograph of the Direct Fluid Injection/Supercritical Fluid Chromatography/Mass Spectrometry Ion Source ............... 
6. Photograph of the Assembled Direct Fluid Injection/Supercritical Fluid Chromatography/Mass Spectrometry Instrumentation, Showing the Interface Probe Ready for Insertion Into the Mass Spectrom-

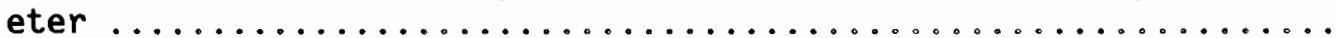

7. Total Ion Current Chromatogram (A) and Negative Ion Mass Spectrum (B) from the Analysis of Perfluoroproprionic Acid-Derivatized Amino-Polynuclear Aromatic Hydrocarbons by Negative Ion Chemical Ionization/Direct Fluid Injection/Mass Spectrometry ...........

8. Calibration Curve for Direct Fluid Injection/Mass Spectrometry

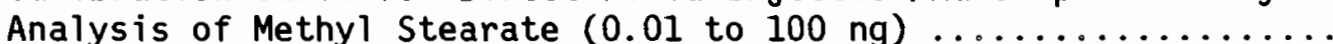

9. Chromatogram of a Mixture of High-Molecular-Weight Polynuclear Aromatic Hydrocarbons Separated by Pentane Supercritical Fluid Chromatography and Monitored with On-Column Fluorescence Detec-

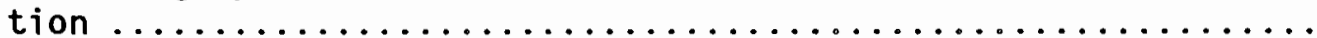

10. Schematic Diagram of Modified Ion Source Region $\ldots \ldots \ldots \ldots \ldots$

11. Schematic Diagram of Power Supply and Potential Control for

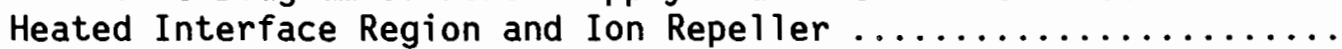

12. Capillary Gas Chromatography Test Chromatogram of a "Polarity

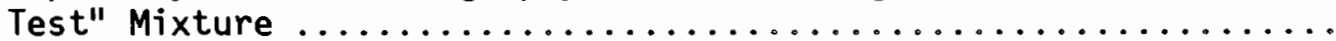

13. Capillary Gas Chromatography Test Chromatogram of a "Polarity

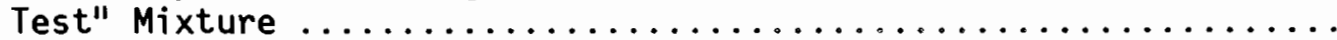




\section{LIST OF ABBREVIATONS}

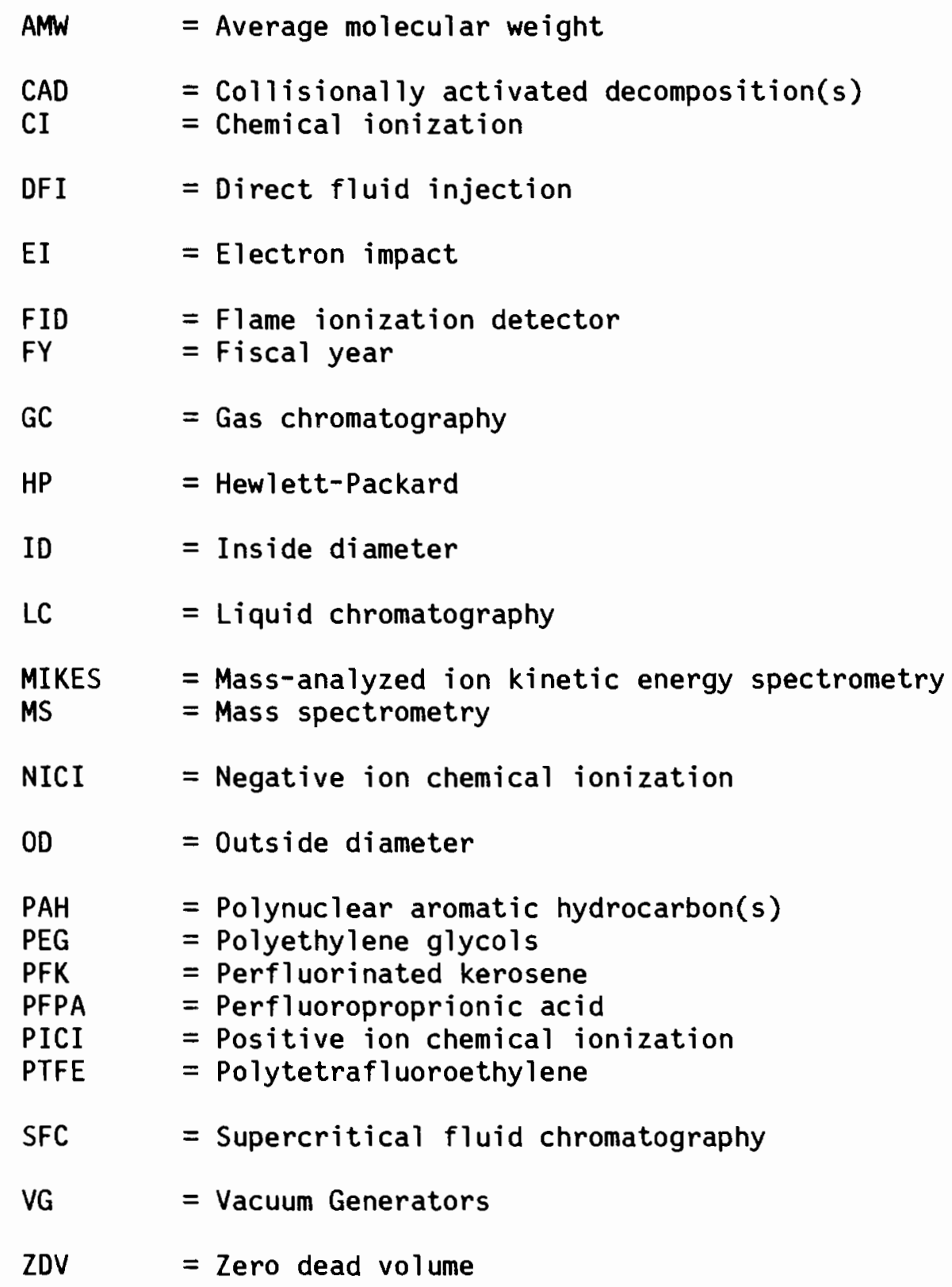




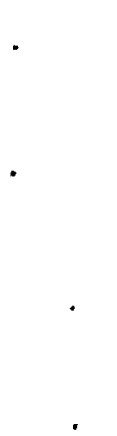




\section{INTRODUCTION AND PROGRAM GOALS}

Modern coal conversion processes have analytical chemistry needs that include the analysis of high-molecular-weight and/or polar organic compounds found in extremely complex mixtures. Analytical techniques capable of such analyses that are also compatible with on-line process monitoring are highly desirable. Current synfuels process monitoring techniques provide information about reaction conditions and bulk characteristics of the process materials, such as temperature, pressure, reactant gas (hydrogen) metering and mass balance. The technologies for measuring other properties, such as viscosity and density, are well-developed in the petroleum-refining industry. Such information gives the process engineer some insight as to how the process is operating in general, but does not provide the detailed chemical information about the process materials that is ultimately required in order to judge how successful the process has been. On-line process monitoring by gas chromatography (GC) is used by the chemical industry, and recentiy in coal liquefaction

processes, for the analysis of gases and relatively volatile chemical components of feed and product lines in production processes that require careful process control. However, the heavy-molecular-weight, relatively nonvolatile components are not monitored in detail in industrial processes due to the lack of appropriate instrumentation.

The goals of this program are to design and develop a combined capillary column supercritical fluid chromatography (SFC)/high-performance mass spectrometry (MS) capable of analyzing high-molecular-weight polar materials and to evaluate the system for application to coal conversion process monitoring. The program involves the development of a new SFC/MS interface capable of allowing direct fluid injection (DFI) and supercritical fluid chromatographic introduction of a wide range of samples into the ion source of a double-focusing, high-resolution mass spectrometer. This program also includes the development of required polar fluids and compatible chromatographic phases for efficient separation and analysis of polar and high-molecular-weight components. We will also evaluate the potential use of such instrumentation in online process monitoring for industrial chemical processes that produce such complex mixtures, including some coal conversion processes. 
The combination of gaseous and liquid properties exhibited by supercritical fluids can be exploited by using supercritical fluids as mobile phases in chromatography and as the transport medium for sample introduction in MS. The DFI process, illustrated schematically in Figure 1, allows material dissolved in a supercritical fluid to be transported to the gas phase for analysis (Smith and Udseth, 1983a). This approach allows relatively high-molecularweight compounds to be analyzed, with detection efficiencies in the picogram range (Smith and Udseth, 1983b). We anticipate that the high chromatographic resolution possible with capillary column SFC will allow the extension of high-resolution MS to the characterization of polar, high-molecular-weight materials that cannot be separated by GC with better separations than are presently possible with liquid chromatography (LC).

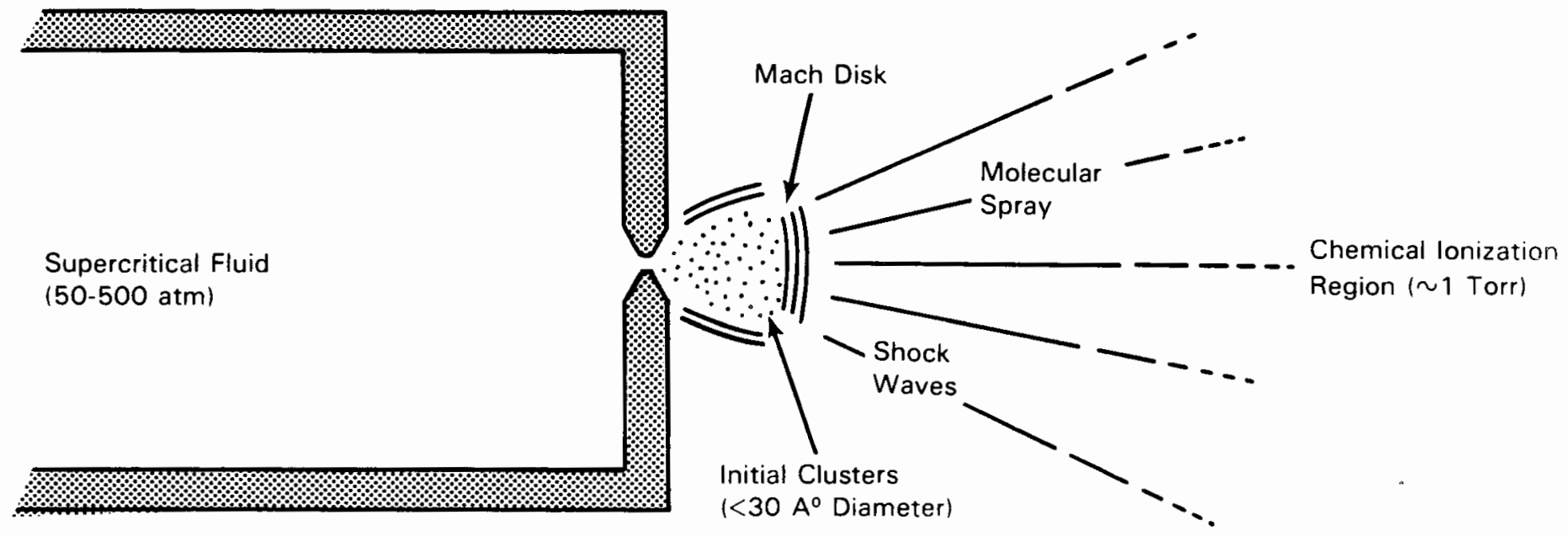

FIGURE 1. Schematic Illustration of the Direct Fluid Injection Process. The Mach disk is the shock wave perpendicular to the expansion axis, resulting from the expanson into the lower pressure region.

Although the term "direct fluid injection" actually refers to a method of introducing a supercritical fluid directly into the ion source of a mass spectrometer, this term has also been associated with the process of transporting a sample into the mass spectrometer, using a supercritical fluid, via a short length of uncoated fused silica capillary tubing. This method is in contrast to using a length of fused silica tubing whose interior surface has been coated with a chromatographic stationary phase. The terms SFC and DFI will be used in this report with these operational definitions to allow distinction 
between experiments involving SFC and those that do not involve DFI chromatographic separation of the analytes during transport to the mass spectrometer. 


\section{TECHNICAL PROGRESS}

The technical progress of the first 18 months of this program, from its initiation in February 1984 to the end of fiscal year (FY) 1985, will be reviewed in this fourth-quarter, Technical Progress Report for FY 85 . The progress achieved will be discussed in general topic areas, not as specific program tasks, as has been the format in previous Technical Reports.

DEVELOPMENT AND EVALUATION OF INSTRUMENTATION

Instrument Development

The approach to developing an integrated SFC/high-performance MS system, complete with a computer-based data system, necessarily consisted of a series of steps, starting with the construction of a suitable interface and modifying the mass spectrometer to accommodate the interface. Our approach was to design the interface and mass spectrometer modifications without interfering with the operation of the mass spectrometer in other modes. We also provided for quick and easy turnover of the instrument for various experimental applications used in other programs, many of which did not involve SFC/MS.

The instrumentation essentially consists of three basic subunits: a solvent delivery system, a supercritical fluid chromatograph, and a mass selective detector (Figure 2). The heart of the solvent delivery system is a Varian 8500 high-pressure syringe pump ( 8000 psi maximum pressure), which delivers a pulse-free flow of pressurized liquid solvent to the chromatograph. The syringe pump is controlled by a microprocessor pressure programmer, which receives input from the syringe pump through a pressure transducer. The highpressure fluid is filtered and cleaned by two $5-\mu \mathrm{m}$-mesh stainless-steel filters before passing through the injector valve and into the chromatograph, where the temperature is maintained above the critical temperature of the fluid.

The supercritical fluid chromatograph/interface consists of a sample injector; a temperature-regulated oven and suboven; a heated interface probe; 


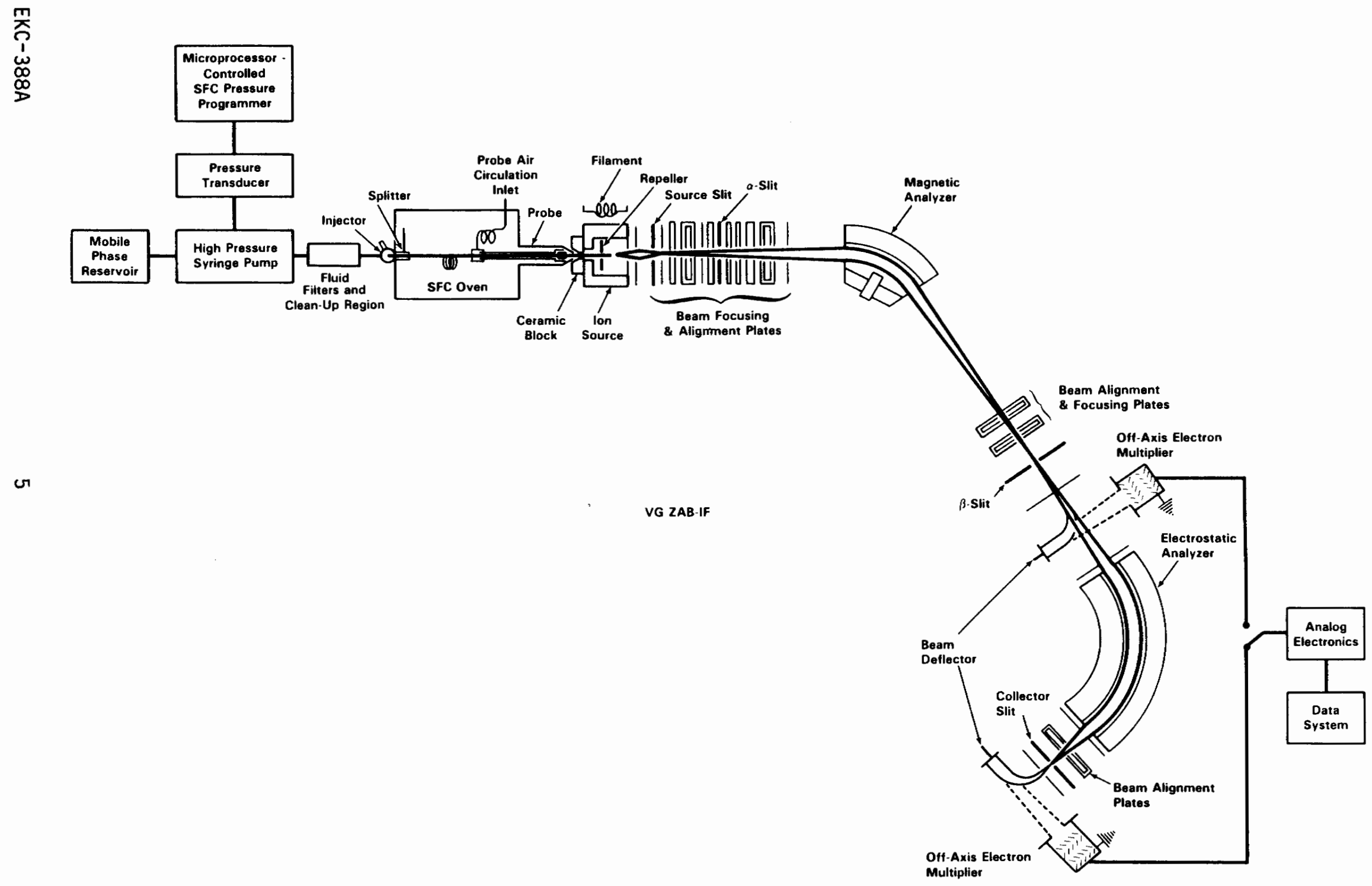

FIGURE 2. Schematic Illustration of the Capillary Column Supercritical Fluid Chromatograph/High-Resolution Mass Spectrometer System 
and a fused silica capillary column, or transfer line, through which the supercritical fluid flows. The syringe pump is connected to a four-port valco ultra-high-pressure LC injector valve by way of heavy-walled 1/16-in.-diameter stainless-steel tubing. The injector valve is mounted atop a modified gas chromatograph oven (Hewlett-Packard [HP], Model 5700) and is fitted with a $0.060 \mu 1$ injection loop. The injector valve is connected to the fused silica capillary column (or transfer line) either directly or via a fluid flow splitter, which allows only a portion of the injected volume to pass into the fused silica column.

The chromatograph oven, suboven, and interface probe are shown schematically in Figure 3. The suboven unit on the gas chromatograph was assembled from asbestos-free refractory sheets, and a baffle was constructed from thin copper sheeting to direct heated air from the main oven into the suboven. Auxiliary heaters, constructed from HP GC injector block heaters, were installed in the suboven to supplement the forced-air heating. The interface probe was constructed from a $\frac{1}{2}-i n$-diameter stainless-steel tube, approximately $48 \mathrm{~cm}$ long. A heater running the length of the probe was made by wrapping a nichrome wire around a ceramic tube, which houses the fused silica transfer line and small thermocouples. The interface probe was connected to the front face of the suboven. Temperature measurements made along the length of the inside of the interface probe indicated that, with sufficient air (preheated in the main oven) blown through the probe, as indicated in Figure $3, a$ uniform temperature $\left(200 \pm 2^{\circ} \mathrm{C}\right)$ could be maintained over the length of the probe. The air flow was necessary to eliminate the thermal gradient inherent in the probe construction.

In the initial interface probe design, the fused silica tubing was connected to the injector valve and was directed through the main oven, the suboven and, finally, through the interface probe, thus serving as the heated transfer line for the supercritical fluid. To limit the pressure drop across

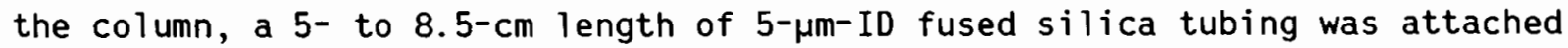
to the end of the 50- $\mu \mathrm{m}$-ID transfer 1 ine to act as a flow restrictor. The union was made with a zero dead volume (ZDV) union (Valco), which was housed in the body of the heated probe. The flow restrictor was sealed in at the probe tip with a Vespel ferrule (Figure 3), thus making the body of the inter- 


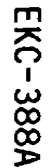

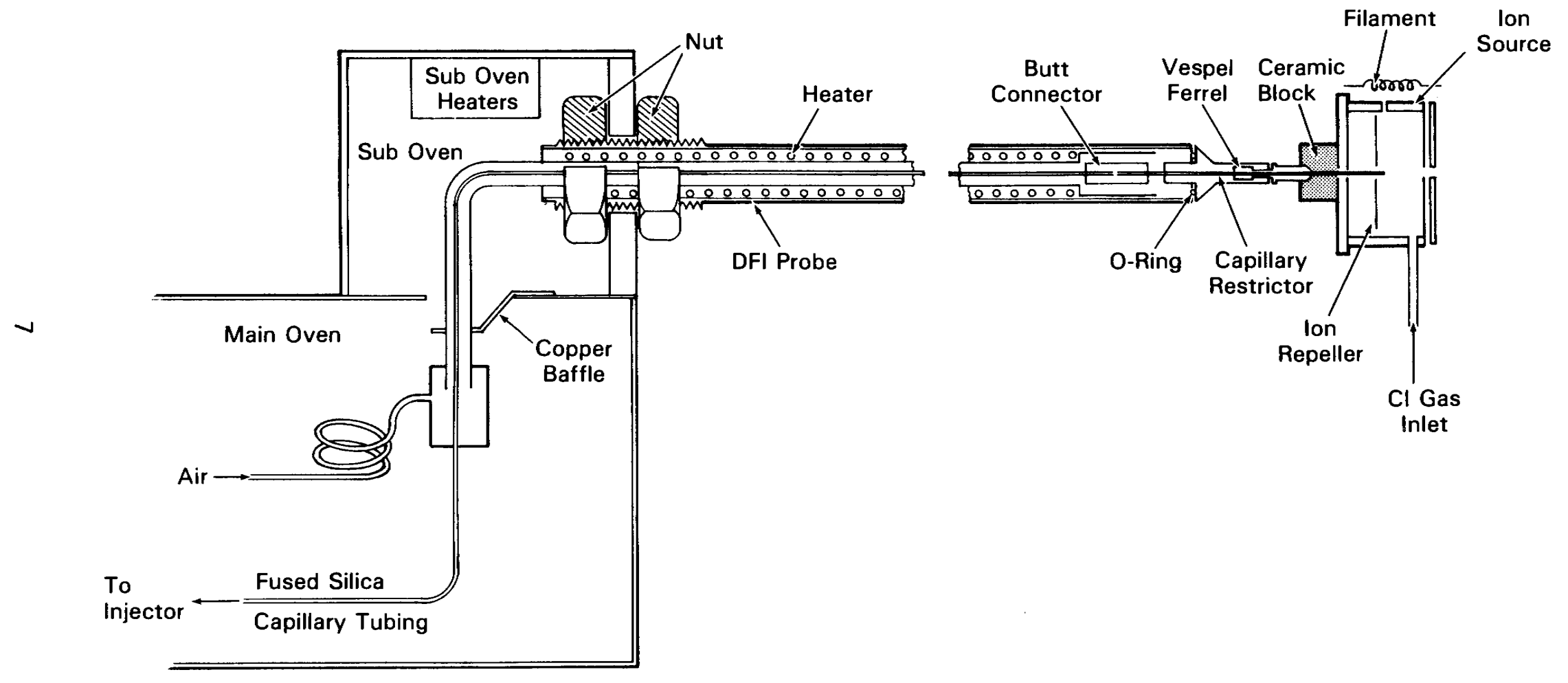

FIGURE 3. Schematic Illustration of the Direct Fluid Injection/Supercritical Fluid Chromatography Oven, Interface Probe, and Ion Source 
face probe vacuum-tight. The flow restrictor protruded from the probe tip to a length that allowed the end of the restrictor to extend into the ion source when the interface probe was mated with the back of the ion source. The probe was later modified to allow a single ZOV butt connector, housed in the probe end cap, to serve as a vacuum seal and to attach the restrictor to the fused silica column (Figure 4).

The stainless-steel ion source was based on the design of a standard Vacuum Generators (VG) electron impact/chemical ionization (EI/CI) ion source so as to be compatible with the instrument's electronics and geometry, but was modified to accommodate the entry of a $\frac{1}{2}-$ in.-diameter probe through the back side of the ion source, co-axial with the center of the ion exit slit. Other modifications to the standard source design included: removal of the electron trap; reduction of the internal volume by one-third; elimination of all unnecessary reentrant holes; and fabrication of a repeller plate with a hole in the center to allow entry into the ion source volume of the flow restrictor from the back side of the ion source. In addition, the ion exit slit was fixed at $0.13 \mathrm{~mm}$ in order to "tighten" the ion source to maintain the relatively high pressures required for optimal CI operation. A ceramic glass block was machined to fit tightly against the cone-shaped tip of the interface probe, and was attached to the back side of the ion source (as shown in Figure 3 ). A photograph of the assembled ion source is shown in Figure 5 .

The mass spectrometer used in this development program was a VG ZAB 1-F, a double-focusing instrument of reverse Nier-Johnson geometry, capable of high mass resolution while operating at good sensitivity, and equipped with a partially laminated magnet having an upper mass limit of 1750 amu at $8000-V$ ion acceleration. The standard $190 \mathrm{~L} / \mathrm{min}$ mechanical roughing pump attached to the source housing diffusion pump was replaced with one rated at $760 \mathrm{~L} / \mathrm{min}$ in order to allow the diffusion pump to handle the larger volumes of gases associated with DFI. A $\frac{1}{2}-$ in. -ID vacuum ball valve and associated vacuum plumbing was attached to a modified vacuum housing end flange containing the 16 electrical vacuum feed-throughs required by the ion source. The interface probe vacuum lock (i.e., the ball valve) and attached end flange are dedicated to SFC/MS applications, and change-over to an experimental mode other than SFC/MS requires only the simple and rapid exchange of end flanges and ion sources. The assembled SFC/interface probe unit was mounted on a laboratory cart, 


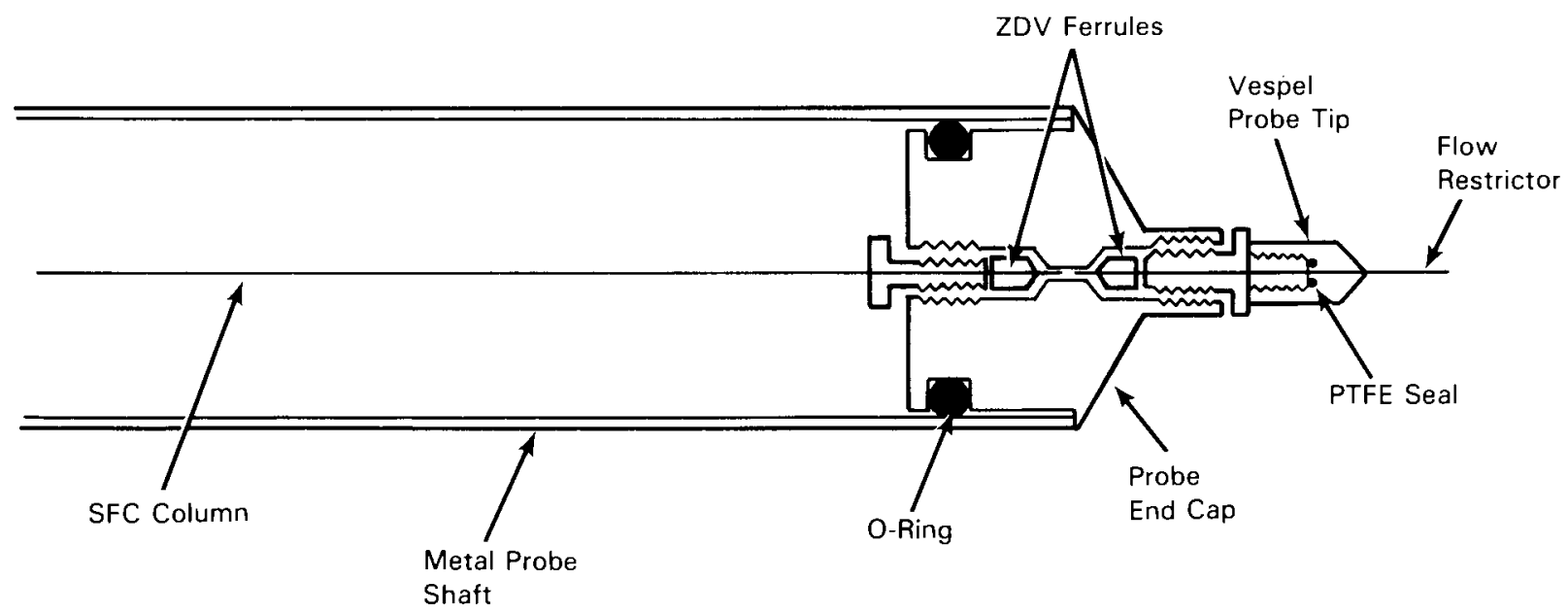

FIGURE 4. Schematic Diagram of Modified Interface Probe

which, in turn, was mounted on a transport platform featuring linear bearings. These bearings allow the cart and instrument to be smoothly transported over a lenth of $53 \mathrm{~cm}$. Thus, once the platform/cart assembly is aligned with the axis of the mass spectrometer, the interface probe can be readily inserted through the vacuum lock into the ion source housing, and accurately and reproducibly mated with the ion source. A photograph of the assembled chromatograph/mass spectrometer is shown in Figure 6 .

\section{Instrument Evaluation and Modifications}

The sequence of investigations for evaluating the performance of the assembled instrumentation was designed to minimize the number of known variables present during any new investigation. Thus, the performance of the newly constructed ion source was first examined without the DFI/SFC interface probe in place. The next evaluation focused on the ion source and interface performance by introducing a supercritical fluid without chromatography (DFI). Sample introduction involving chromatographic separation of analytes (SFC) has recently been introduced in the evaluation program. During the evaluations, various deficiencies in design or performance have been detected, and modifications have been made to the instrumentation to improve or correct the problems. The development of the instrumentation has proceeded in a stepwise, 


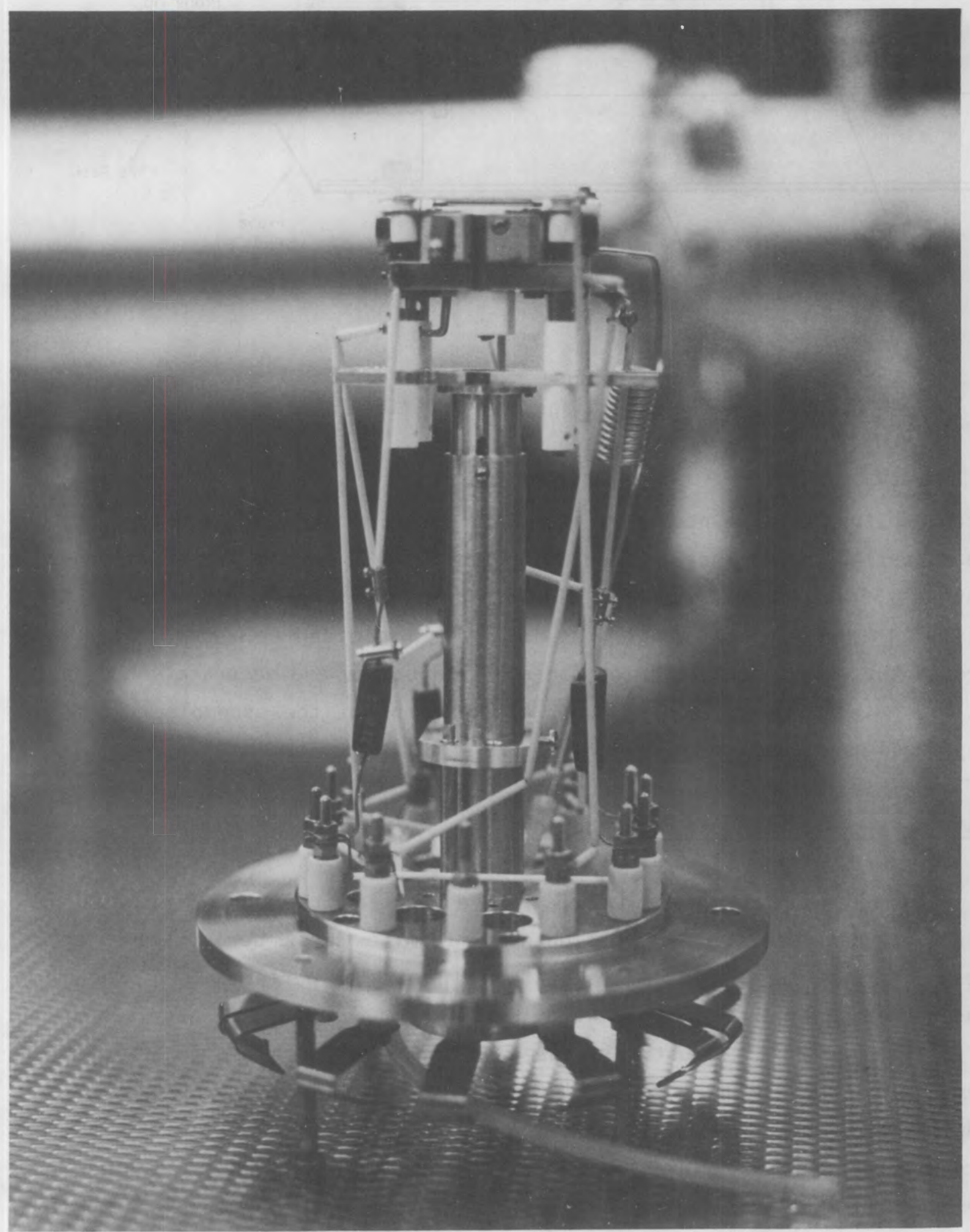

FIGURE 5. Photograph of the Direct Fluid Injection/Supercritical Fluid Chromatography/Mass Spectrometry Ion Source 


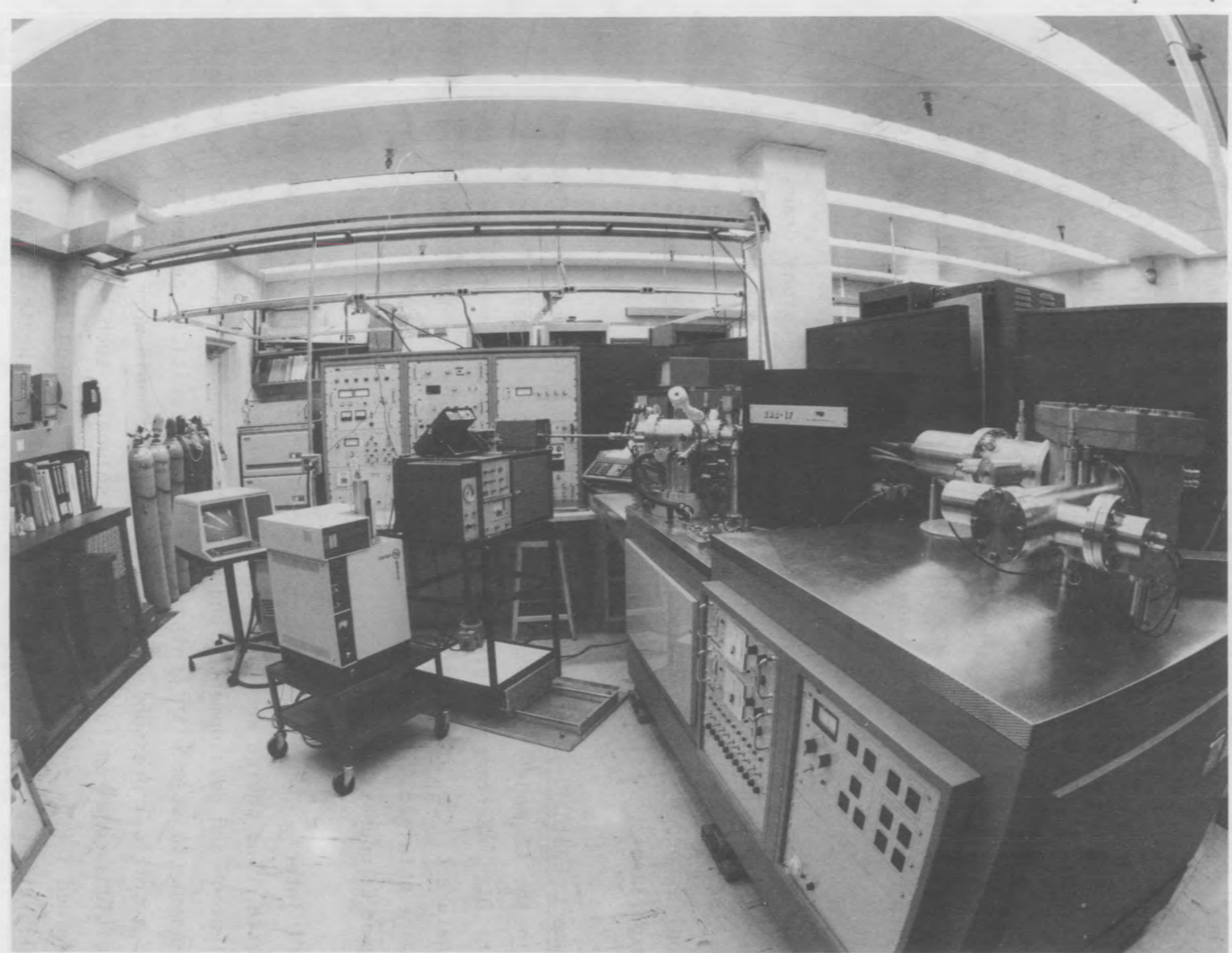

FIGURE 6. Photograph of the Assembled Direct Fluid Injection/Supercritical Fluid Chromatography/Mass Spectrometry Instrumentation, Showing the Interface Probe Ready for Insertion Into the Mass Spectrometer 
interactive fashion, so that each new experiment provides data that can be used to improve instrument performance.

Two other areas of research related to the program goals have been under investigation concurrently with the instrumentation development. These involve development of the required polar fluid phases and compatible chromatographic stationary phases needed for efficient separation of polar compounds by SFC, and the development of improved fluid flow restrictors. The ultimate successful analysis of high-molecular-weight, polar chemicals depends as much on the development of these two areas as on the instrumentation development. The research conducted on capillary column development and fluid flow restrictor fabrication will be discussed separately following this discussion section.

The results of the evaluation experiments and conclusions drawn from these results will be discussed independently from some of the modifications made to the instrumentation. The following discussions will proceed roughiy in chronological order, but no attempt has been made to follow the exact sequence of experiments.

Instrument Evaluation. The newly assembled ion source was tested for operation in the CI mode, using methane as the CI reagent gas. The entrance hole for the DFI interface was plugged for these experiments to maintain the gas-tight nature of the ion source. Satisfactory ratios for the methane reagent ions were obtained, indicating that the ion source was sufficiently gastight for good CI operation.

The DFI interface and chromatograph were next tested in operation with the ion source. The choice was made to use pentane as the supercritical fluid in the evaluation and development program on the assumption that, if the system coud be made to work with pentane, which has a critical temperature of $196.6^{\circ} \mathrm{C}$ and a critical pressure of $33.3 \mathrm{~atm}$, then it would work under the less severe conditions required for other nonpolar solvents, such as $\mathrm{CO}_{2}$. The materials examined in the initial DFI experiments were chosen for potential use as mass calibration standards, which were needed to calibrate the mass scale for computerized acquisition of data. Materials examined included perfluorin- 
ated kerosene (PFK), a mixture of halogenated aromatic hydrocarbons, polystyrene, perfluorinated alkyl ethers (Fomblin oils), perfluoroalkyl phosphazines, and polyethylene glycols (PEG). Various methods of introducing these materials into the ion source were investigated. The best results were obtained by placing the calibration standard in a small glass capillary, which was then inserted in the ion source on the end of a direct insertion probe.

Accurate mass calibration files were written and stored in the data system, which allow computer-acquired mass spectra of these calibration standards to be used to generate time-to-mass conversion scales. These scales are used by the computer to assign masses to spectra acquired in a time-versus-intensity format. PFK and Fomblin oils gave the best results for EI and negative ion chemical ionization (NICI) modes of ionization. Fomblin oil was used under EI conditions to calibrate the data system to $2000 \mathrm{amu}$, the upper mass range limit for the present data system. The PEG samples have proven to be the best calibration standards for positive ion chemical ionization (PICI) but have not allowed calibration higher than $1000 \mathrm{amu}$. This mass range has been sufficient, thus far, for most routine experiments.

Results from initial DFI experiments ascertained that the flow of pentane into the ion source was sufficient for optimum PICI operation when the pressure on the fluid was maintained above $100 \mathrm{~atm}$. Below this presssure, additional reagent gases, such as methane or isobutane, could be added to modify and optimize the CI conditions. These auxiliary reagent gases had little effect on the sensitivity or specificity of relatively nonpolar analytes, however, and have not been used routinely during instrument development. These initial experiments were conducted using an injector loop volume of $0.2 \mu 1$ and without any solvent splitting. This resulted in a large solvent plug containing the solutes of interest that moved through the transfer line. When solvents other than pentane were used, e.g., benzene, the ion chemistry of the solvent so dominated the PICI processes in the ion source that the solute molecules were not detected with good sensitivity. Two solutions to this problem were investigated: the use of NICI and the use of low-resolution chromatography to separate the solvent from the solutes. 
Although NICI is not universally applicable and works best for analytes containing electronegative elements such as halogens, it does provide sensitive and specific detection of suitable analytes. For example, a mixture of perfluoroproprionic acid (PFPA) amide derivatives of amino-polynuclear aromatic hydrocarbons (PAH) were analyzed by NICI/DFI/MS, using benzene as the solvent. The results (shown in Figure 7) indicate very little contribution from ion source background and no contribution from the internal ion chemistry of benzene. The total ion current chromatogram (Figure 7A) depicts a typical elution profile for a pentane DFI experiment conducted at $150 \mathrm{~atm}$ and $220^{\circ} \mathrm{C}$. The DFI peak is $17 \mathrm{sec}$ wide at half-height, and exhibits some tailing due to adsorptive effects. The mass spectrum (Figure $7 B$ ) shows the coelution of a number of PFPA derivatives, each characterized by a base peak (largest ion in the mass spectrum of an individual compound) at 20 amu less than the calculated value, indicating the loss of HF from the molecular ion.

We also investigated the problem of interferences derived from the presence of an excess of solvent, using a short capillary column coated with a crosslinked octyl polysiloxane stationary phase as the DFI transfer line. By using flow rates greatly in excess of the flow rate typically used for optimum chromatographic performance, the column was used to separate the solvent from the analytes but allow the relatively short analysis time associated with a DFI experiment. The quantitative capability of the DFI/MS instrumentation was examined using this short column. Selected ion monitoring experiments determined low picogram detection limits for methyl stearate. The calibration curve plotting the $\log$ of the area of the signal arising from the protonated molecular ion of methyl stearate versus the gram quantity injected (Figure 8) was linear over five orders of magnitude, with a detection limit of about 10 pg. When the electron multiplier gain of the mass spectrometer was increased so that the higher concentrations were no longer on scale, the detection limit was lowered to less than a picogram, and the response remained linear over three orders of magnitude.

Studies designed to evaluate the performance of the flow restrictor and interface design were conducted with monodispersed polymers whose oligomer distributions were well known. These polymers included polystyrene (average molecular weight [AMW], $800 \mathrm{amu}$ ) and polybutadiene. The polymers were an- 

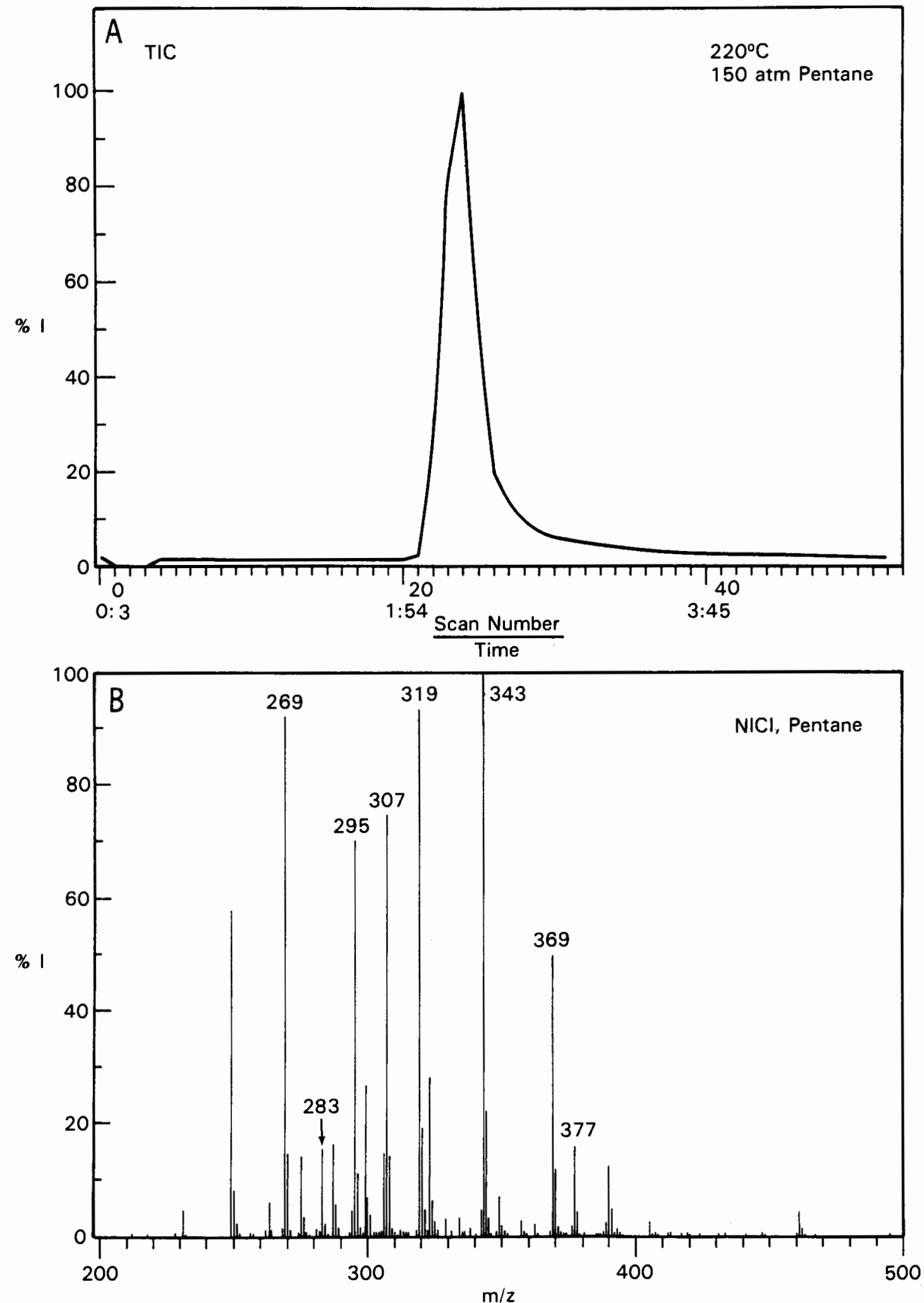

FIGURE 7. Total Ion Current Chromatogram (A) and Negative Ion Mass Spectrum (B) from the Analysis of Perfluoroproprionic Acid-Derivatized Amino-Polynuclear Aromatic Hydrocarbons by Negative Ion Chemical Ionization/Direct Fluid Injection/Mass Spectrometry 


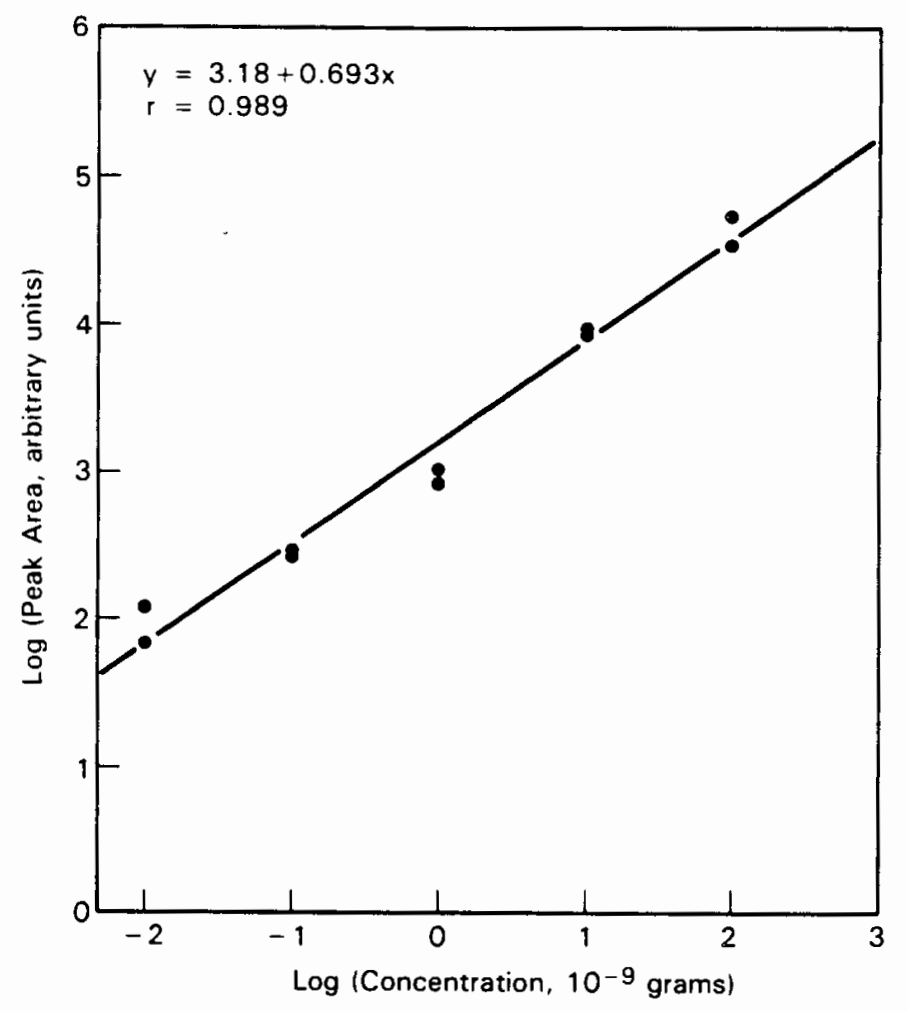

FIGURE 8. Calibration Curve for Direct Fluid Injection/Mass Spectrometry Analysis of Methyl Stearate (0.01 to $100 \mathrm{ng}$ )

alyzed by direct-probe PICI, using methane and pentane as reagent ion gases. These results were compared to those from DFI analyses using supercritical pentane. The spectra were very similar from these two analyses (masses were observed out to $1200 \mathrm{amu}$ ), but the relative oligomer distribution observed in both types of analyses did correspond well to the reported oligomer distributions. Further studies using mixtures of $P A H$ ranging in size from three to seven rings also indicated that a mass discrimination against higher-molecular-weight, lower-volatility components occurred somewhere in the DFI process.

Polystyrene oligomers with molecular weights in excess of 3500 amu have been chromatographed using supercritical pentane and detected with in-1ine ultraviolet-visible spectrophotometric detectors (Fjeldsted et al., 1983). Mixtures of $\mathrm{PAH}$, some containing even heavier-molecular-weight components than were used in the DFI/MS experiments, were analyzed in our laboratory by SFC, using on-column fluorescence detection. PAH as large as coronene, decacyc- 
lene, and rubrene were readily chromatographed using supercritical pentane, and detected on-column just prior to exiting the column through the flow restrictor (Figure 9). We therefore concluded that although the high-molecularweight material was being transported to the end of the column, it was not passing through the flow restrictor used in the DFI/MS experiments. These results clearly indicated that a better understanding of the problem was needed that would lead to modifications to the interface and flow restrictor.

SFC experiments conducted with a flame ionization detector (FID) showed that the restrictor discrimination against higher-molecular-weight materials decreased as the temperature of the FID increased. Modifications were made to the ion source to allow the last $1.5 \mathrm{~cm}$ of the flow restrictor to be heated independently of the ion source to temperatures $>450^{\circ} \mathrm{C}$. A schematic diagram (not to scale) of the modified region of the ion source is shown in Figure 10. A thin-walled, stainless-steel tube was spot-welded into the hole in the ion repeller. A ceramic sleeve electrically isolates the modified repeller from the ion source back plate, through which the thin-walled tube passes out of the ion source volume. An electrical connection was made to the end of the metal tube with a brass collar and an attached copper wire. Another electrical connection was made to the modified repeller through one of two support legs of the repeller (not shown in the diagram), which also passes through the ion source back plate and through the ceramic probe adapter block. The support legs were threaded, and the ceramic block was bolted against the ion source back plate, using the threaded legs and two small nuts. The ceramic block provides electrical isolation between the ion source and the Vespel (DuPont) probe tip. Because the fused silica restrictor must pass through a very small hole in the ceramic block (necessary to reduce the flow of ionized gas out of the ion source), a small metal guide, which functions like a funnel, was used to direct the restrictor into the hole.

Because the heating region has been made an integral part of the ion repeller, the power supply for the heater and all electrical connections had to be maintained at the normal ion repeller potential, which is typically within $\pm 2 \mathrm{~V}$ of the ion source potential (full accelerating potential) up to $6000 \mathrm{~V}$. This required that the heater power supply be electrically isolated from the grounded building power supply through the intervention of an isolation trans- 


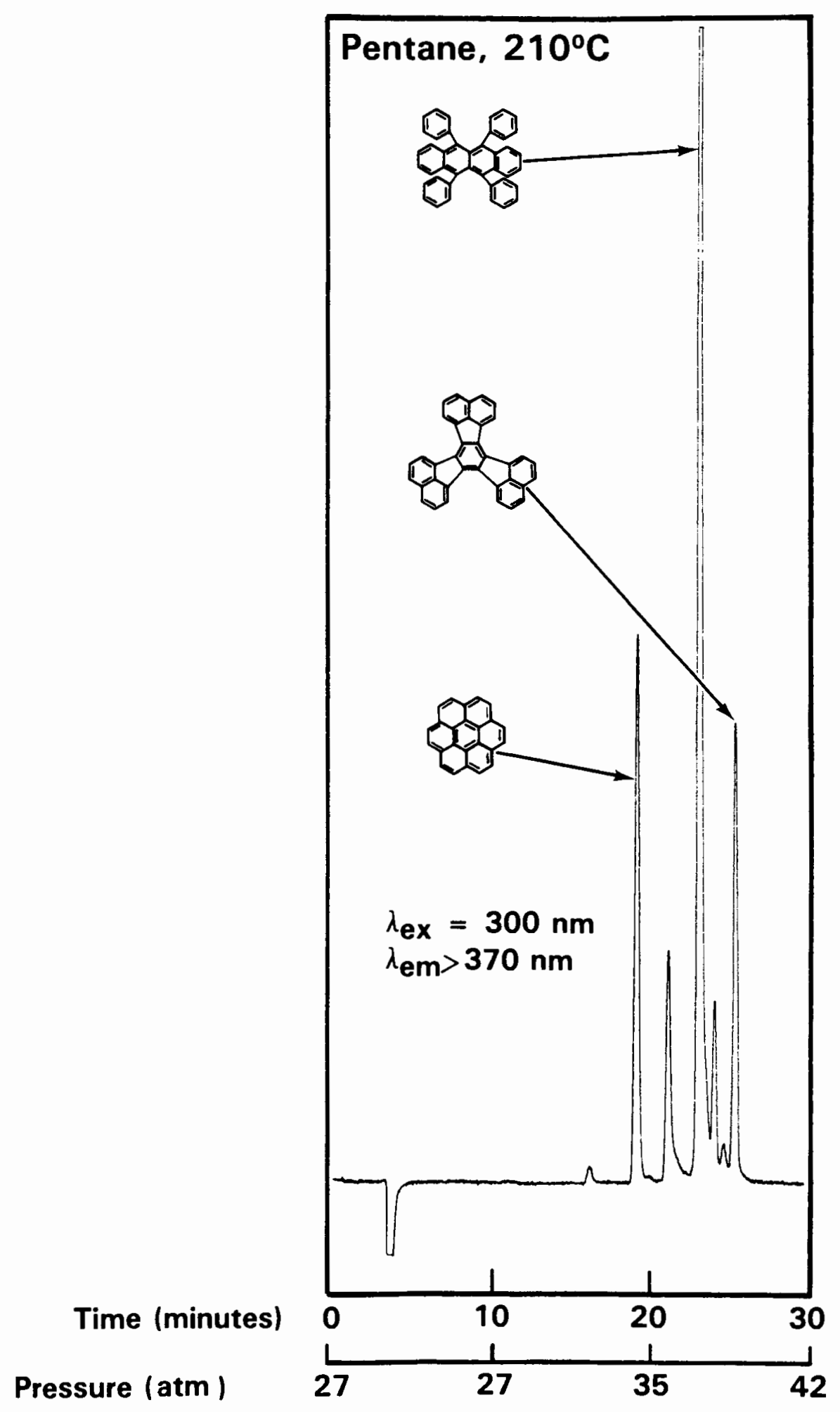

FIGURE 9. Chromatogram of a Mixture of High-Molecular-Weight Polynuclear Aromatic Hydrocarbons Separated by Pentane Supercritical Fluid Chromatography and Monitored with On-Column Fluorescence Detection 


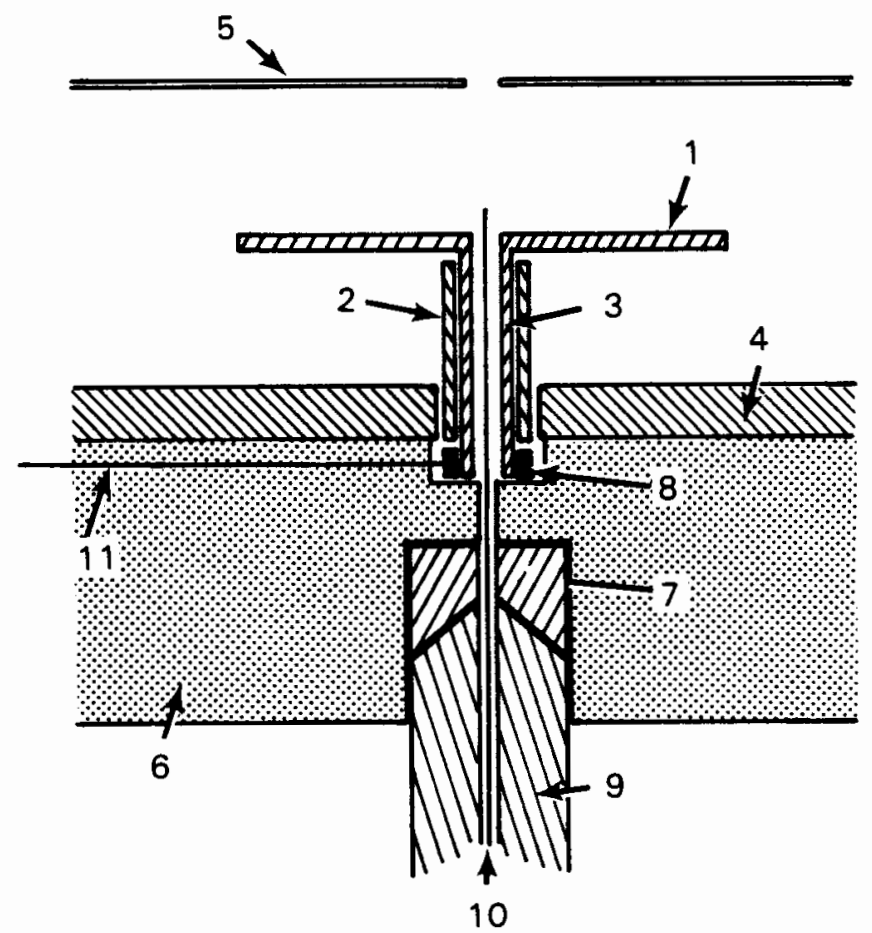

FIGURE 10. Schematic Diagram of Modified Ion Source Region

1. Ion repeller plate

2. Ceramic sleeve

3. Thin-walled stainless-steel tube

4. Ion source back plate

5. Ion source exit plate

6. Ceramic adapter block
7. Metal guide

8. Collar electrode

9. Interface probe tip

10. Fused silica capillary restrictor

11. Copper wire lead

former, and that the electrical potential of the power supply be determined by the ion source repeller offset control. This arrangement is shown schematically in Figure 11.

In initial tests with the modified interface region, an $8-\mathrm{cm}$ length of 12.5- $\mu$ m-ID capillary tubing was used as the capillary flow restrictor. A mixture of PAH containing components with molecular weights in the range of 128 to 300 amu was analyzed by DFI/MS, using isopentane as the supercritical fluid at $220^{\circ} \mathrm{C}$ and at pressures over the range of 30 to $60 \mathrm{~atm}$. In addition, a mixture of polystyrene oligomers (AMW, 800) was analyzed under the same conditions. No decrease in mass discrimination for either sample was observed as the temperature of the heating region was varied from 220 to $600^{\circ} \mathrm{C}$. However, 


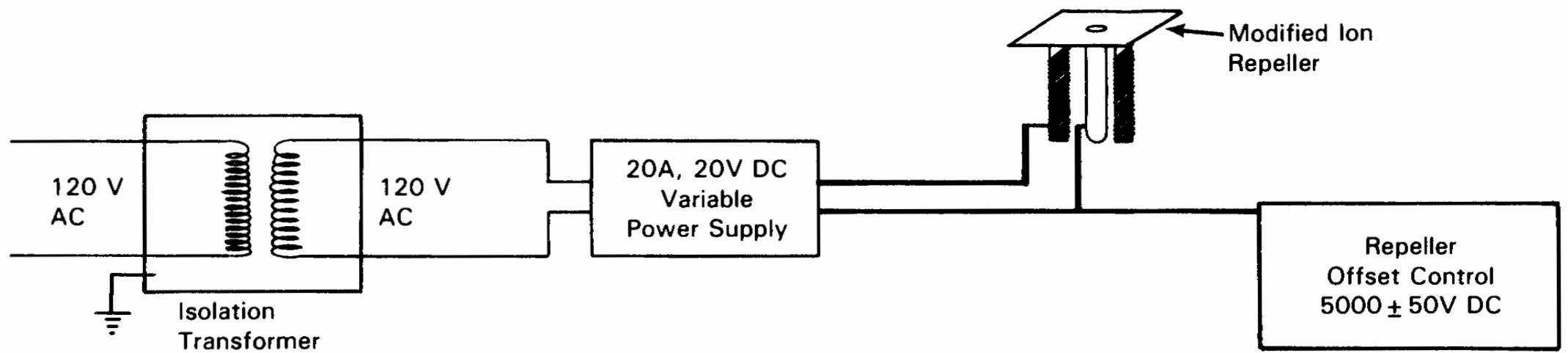

FIGURE 11. Schematic Diagram of Power Supply and Potential Control for Heated Interface Region and Ion Repeller 
because heating occurred only over the last $1.5 \mathrm{~cm}$ of the total restrictor length of $8 \mathrm{~cm}$, the results of these experiments were not conclusive.

Similar experiments were performed using a drawn restrictor, in which the entire length of restriction in the tubing was contained in the heated region. Although no measurable decrease in discrimination was apparent, dramatic changes in ion source pressure were observed as the temperature was varied. As the temperature increased, the density of the supercritical fluid rapidly decreased, thus decreasing the mass flow into the ion source, resulting in lower pressures and decreased overall sensitivity. Thus, even if the mass discrimination was improved, it was not clear that we would have detected a small change. Further investigations with the heating region will be required.

In order to investigate whether the higher mass discrimination could be affected by changing the rate of flow through the capillary restrictor, the ion source was modified to allow additional pumping within the ion source block. The source housing vacuum system was not designed to handle the higher gas loads that these investigations would produce. Therefore, modifications were necessary if increased flow rates, arising from higher pressures of supercritical fluid and/or from capillary flow restrictors with larger internal diameters, were to be investigated.

A $\frac{1}{4}$-in.-diameter hole was drilled in the side of the ion source, and a Teflon tube (0.75-in. OD, 0.625-in. ID) was attached to this hole via a short length of stainless-steel tubing. The Teflon tube was attached to a $10-\mathrm{L} / \mathrm{sec}$ mechanical pump via an 0.75-in.-ID vacuum hose. A high-conductance valve was placed in the vacuum line to allow control of the amount of pumping on the source by the rotary pump. With the valve fully open, the pressure inside the ion source, as measured by a capacitance manometer, was reduced by a factor of three compared to the pressure measured with the valve fully closed. Thus, this arrangement allowed us to admit a minimum of twice the volume of supercritical fluid per unit time as could be admitted without the extra pumping. It was quickly determined that the pressure of gas within the tubing leading from the ion source to the roughing pump was ideal for supporting an electri- 
cal discharge from the ion source to the mechanical pump when the ion source was operated at $3000 \mathrm{~V}$ or greater potential.

Operating the ion source at an accelerating potential of $2000 \mathrm{~V}$, a series of experiments were conducted to determine if the mass discrimination problem improved with the higher flow rates and to determine the loss of sensitivity due to the extra pumping. For the same amount of fluid entering the source, the instrument was a factor of approximately 2.5 times more sensitive when the extra source pumping was not in use. Since the pressure in the ion source dropped by a factor of about 2.5, it appeared that the loss in sensitivity was directly proportional to the amount of sample lost through pumping, exactly as predicted. The results from experiments using mixtures of PEG to examine the mass discrimination phenomenon were less instructive, however, indicating only a small improvement in mass transport through the flow restrictor. The loss of sensitivity experienced in these experiments propably did not allow an accurate assessment to be made. Further investigations are planned.

Initial SFC/MS experiments were conducted using a $9-m, 50-\mu m-I D$, fused silica capillary column that was coated internally with a crosslinked, deactivated stationary phase of an octyl polysiloxane polymer. When pentane was used as the supercritical fluid at 50 atm and $220^{\circ} \mathrm{C}$, a mixture of $\mathrm{n}$-alkanes ( $C_{12}$ to $C_{21}$ ) coeluted with the solvent peak (hexane) and showed no separation. Results were similar for a coal-tar sample. These results indicated that the $50 \mathrm{~atm}$ pressure was too high, resulting in a supercritical fluid of sufficient density and solvating power that the analytes were not retained in the column stationary phase to any greater extent than the solvent. When the pressure was lowered to $35 \mathrm{~atm}$, some separation among the analytes was realized, but the large amount of solvent present $(0.2 \mu 1$ injection volume) made the detection of the early eluting analytes difficult. In addition, the pentane fluid was modified by the solvent plug to create a more-polar solvent, leading to decreased retention of the analytes. The problem of excess solvent was addressed by installing a fluid flow splitter on the injector valve, and by decreasing the injection volume to $0.060 \mu \mathrm{l}$. Isobaric analysis of the coal-tar mixture at $220^{\circ} \mathrm{C}$ and $35 \mathrm{~atm}$, with a split ratio of $10: 1$ (ten parts rejected: one part analyzed), resulted in separating the solvent from the mixture component and partial resolution of the analytes. SFC/MS analysis of polystyrene 
(AMW, 800) was carried out at $220^{\circ} \mathrm{C}$ and $30 \mathrm{~atm}$. The largest oligomer that eluted had a molecular weight of $684 \mathrm{amu}$, consistent with a mass discrimination against the higher-molecular-weight components, which were more abundant than the lower-molecular-weight species in this mixture, and actually plugged the capillary restrictor during several experiments.

Other Instrument Modifications. Considerable effort has been spent in refining the design of the interface region so as to allow routine operation of the ion source at accelerating potentials as high as $6000 \mathrm{~V}$. The design detailed in Figure 4 respresents the latest version. Important operational features of this design include the Vespel probe tip, which supplies the additional electrical resistance found necessary between the metal restrictor guide and the metal body of the probe, and the polytetrafluoroethylene (PTFE) seal around the restrictor, which isolates the metal probe from exposure to the CI plasma inside the ion source. Refinements such as these have increased the maximum accelerating potential from 2000 to $6000 \mathrm{~V}$.

During the last quarter of $F Y 85$, the VG $Z A B-1 F$ was modified by adding a collision cell and additional pumping capacity in the second field-free region (the region between the magnetic analyzer and the electrostatic analyzer) of the mass spectrometer. (The precise location is between the beam alignment and focusing plates and the $\beta$-slit shown in Figure 2.) This modification allows structural information to be obtained on molecular ions from components in complex mixtures. This information can be gained by using the magnet to select an ion of interest and colliding this ion with neutral helium gas molecules in the collision chamber, which induces the ion to fragment. The fragments are then analyzed, using the electrostatic analyzer to produce a spectrum that is characteristic of the structure of the mass selected ion. These "MS/MS" spectra can be interpreted in much the same manner as a mass spectrum for structural information, and/or can be compared to spectra derived in the same manner from ions with the same molecular weight and known structural features. The technique of using collisionally activated decompositions (CAD) in association with mass-analyzed ion kinetic energy spectrometry (MIKES) is a well-known method for deriving further information about the identities of components in complex mixtures (McLafferty, 1983). 
Capillary column SFC is similar to other chromatographic separation methods. The chromatographic columns used for both capillary column SFC and capillary column GC generally consist of a length of fused silica tubing ( 2 to 20 $m$ for SFC; 5 to $60 \mathrm{~m}$ for GC), typically $250-\mu \mathrm{m}-I D$ for $\mathrm{GC}$ and $25-$ to $50-\mu \mathrm{m}-I D$ for SFC. The exterior of the tubing is coated with a polyimide polymer to protect the silica tubing against breakage from routine handing. The interior surface of the tubing is coated with a chromatographic stationary phase. The differential partitioning of different solute molecules between the chromatographic mobile phase and this stationary phase results in the physical separation of the different solute molecules. Thus, in both GC and SFC, a mixture of solute species is injected into the mobile phase stream and is transported, via the mobile phase flow, through the column, where the various solute species are separated. The physically separated solutes then exit the chromatographic column and enter some type of detector, where the solute molecules are differentiated from the mobile phase species.

Because one of this program's goals is to use SFC and SFC/MS to analyze polar and high-molecular-weight components from coal conversion processes, a necessary area of research has been to support the development of capillary column technology that can be used to analyze these types of chemicals. The preparation of capillary columns coated with stable films of stationary-phase polymers that remain homogeneous and intact relative to the solvating influences of supercritical mobile phases represents a significant challenge over and above the challenges encountered in preparing high-performance columns suitable for GC (Kong et al., 1984). In general, higher temperatures (e.g., $>150^{\circ} \mathrm{C}$ ) and/or higher-polarity supercritical fluid systems are more deleterious to the stationary-phase coatings than lower temperatures and/or nonpolar fluid systems. The primary approach taken thus far to create capillary columns for use in SFC has been to crosslink and bond the stationary-phase polymer to the silica surface of the column. The specific mechanisms of this approach have involved: 1) modification of the silica surface of the column in preparation for deactivation; 2) deposition of deactivation layers that are bonded to the silica surface, thus providing sites for crosslinking to the stationary-phase polymer; 3) internal crosslinking of the stationary-phase 
polymer itself; and 4) using various techniques to maximize the total amount of crosslinking.

Modification of Silica Surface for Deactivation

Stationary-phase stability is increased when it is crosslinked to a deactivation layer that has been bonded to the silica surface (Wright et al., 1982a). Methods of deactivation that provide a more complete and dense surface layer not only effectively shield the silica surface but also provide maximum crosslinking sites for the stationary phase. Fused silica has a much lower density of surface hydroxyl groups than either glass or porous silica (Wright et a1., 1982b). Consequently, the number of reactive attachment sites per unit area is smaller, and the number of possible surface bonds between the silica and the deactivation layer molecules is less for fused silica compared to the other two surfaces. It seems logical that a higher degree of stationary-phase stability could be achieved if a larger number of bonds to the silica surface could be obtained.

To test this hypothesis, a series of columns were treated with water at several temperatures, rinsed with methanol, and dried, using a standard $200^{\circ} \mathrm{C}$ thermal treatment. The columns were then deactivated and crosslinked, using optimized treatment conditions. Chromatographic evaluations of columns prepared using these treatments were conducted. Figure 12 shows a test chromatogram obtained from a column prepared with a mild, surface-hydroxylation treatment, followed by surface dehydration, surface deactivation with polymethylhydrosiloxane (Woolley et al., 1984), coating with SE-54 stationary phase, and in situ crosslinking of the stationary phase with azo-t-butane (Wright et al., 1982a). The compounds in this "polarity test" mixture were eluted with symmetrical, sharp peaks, indicating that both good deactivation and efficient coating were obtained. Washout data, in which the capacity ratios of several alkanes were measured before and after conditioning with liquid methylene chloride and supercritical $\mathrm{CO}_{2}$ /methanol (80:20) indicated that approximately $5 \%$ of the phase was lost. In general, a small amount ( 1 to $5 \%$ ) of stationary phase is lost due to the presence of undesired, short polymer chains that do not crosslink efficiently. Complete removal of these impurities from the stationary phase before coating and crosslinking is difficult. This column-prep- 


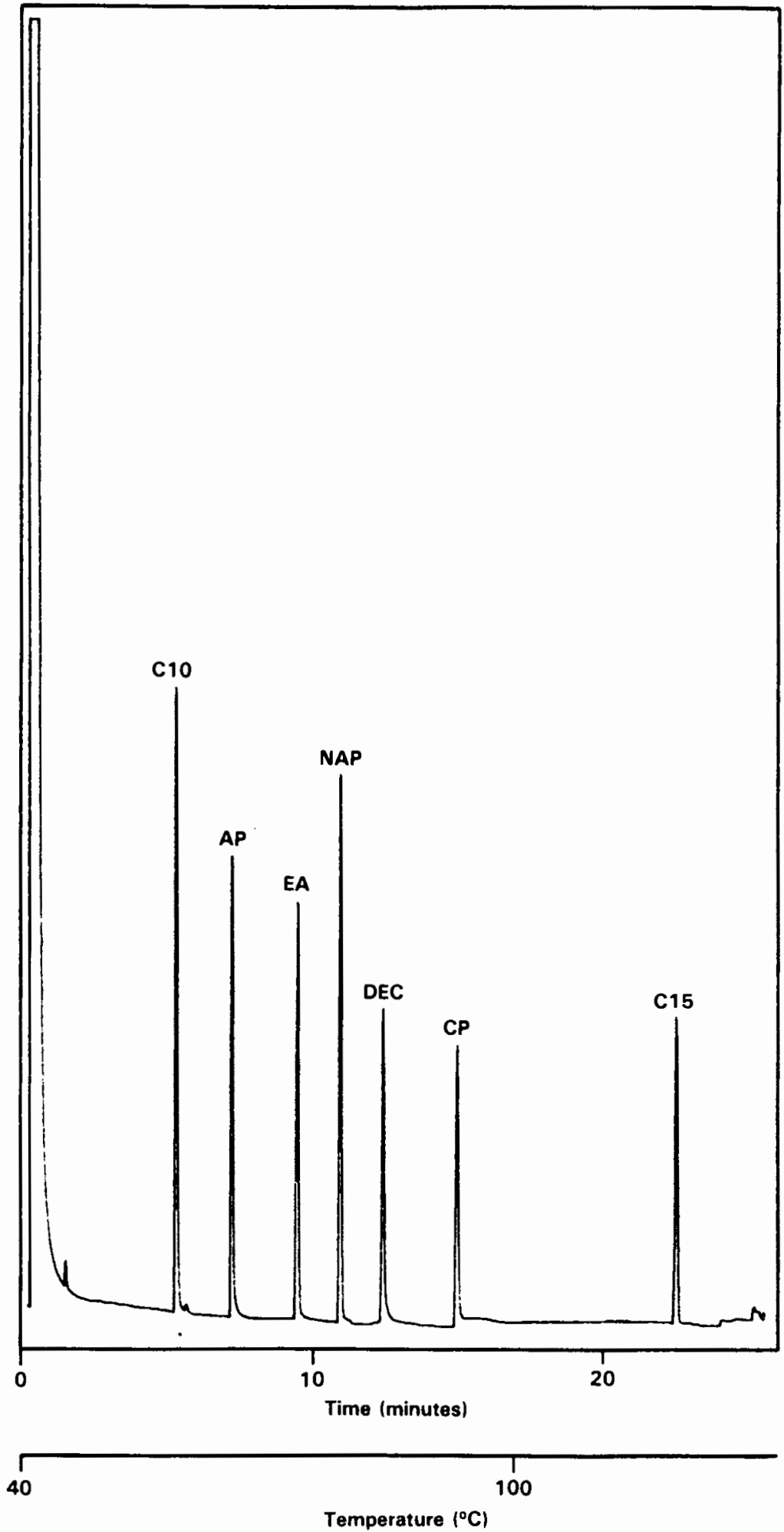

FIGURE 12. Capillary Gas Chromatography Test Chromatogram of a "Polarity Test" Mixture. Conditions: $6-\mathrm{m} \times 50-\mu \mathrm{m}$ column coated with SE-54, temperature-programmed at $4^{\circ} \mathrm{C} / \mathrm{min}$ from $40^{\circ} \mathrm{C}, 300$ psi He carrier gas. Peak identifications: $C 10=\underline{n}$-decane, $A P=$ acetophenone, $E A$ $=$ N-ethylaniline, $\mathrm{NAP}=$ naphthalene, $\mathrm{DEC}=1$-decanol, $\mathrm{CP}=\mathrm{P}^{-}$ chTorophenol, $\mathrm{Cl} 15=\underline{n}$-pentadecane. 
aration procedure appeared very promising for obtaining deactivated, efficient and more stable capillary columns.

In contrast, a chromatogram obtained from a column prepared in a similar manner to that discussed above, but with a harsh surface hydroxylation treatment, is shown in Figure 13 . The same test mixture was chromatographed, and the drastic difference in column quality is evident in the tailing peak shapes. High surface hydroxylation and water content allowed a porous deactivation layer to form, providing a surface that was neither inert nor suitable for highly efficient coating. Thus, this set of experiments demonstrated that milder surface hydroxylation conditions produced a better-performing column, and these milder conditions have been adopted in the silica surface preparation procedure.

Developing a Deactivation Layer

The major emphasis in this phase of the research has been to provide suitable crosslinking sites, centering around a polymethylhydrosiloxane treatment (Woolley et al., 1984). This procedure has several advantages over more traditional silylation treatments and thermally induced rearrangements of monomeric cyclic siloxanes (Stark et al., 1980). For example, hydrosilanes have higher reactivity for bonding to the silica surface hydroxyl groups. Their polymeric nature also maximizes the number of bonded methyl groups per hydroxyl bonding site, which is important because fused silica has a minimal number of surface hydroxyl groups. Consequently, a dense layer of methyl groups is bonded to the silica surface through polysiloxane chains. Treatment and reaction parameters have been investigated and correlated with chromatographic performance to define the optimum conditions for both surface inertness and washout stability. It has also been necesssary to perform a traditional silylation reaction with chlorotrimethylsilane to eliminate any residual activity. This reaction caps any hydrolyzed hydride goups or other remaining hydroxyl functionalities residing in the deactivation layer after surface bonding. This procedure has been optimized and provides a reliable method of producing an inert layer suitable for crosslinking to nonpolar stationary phases. 


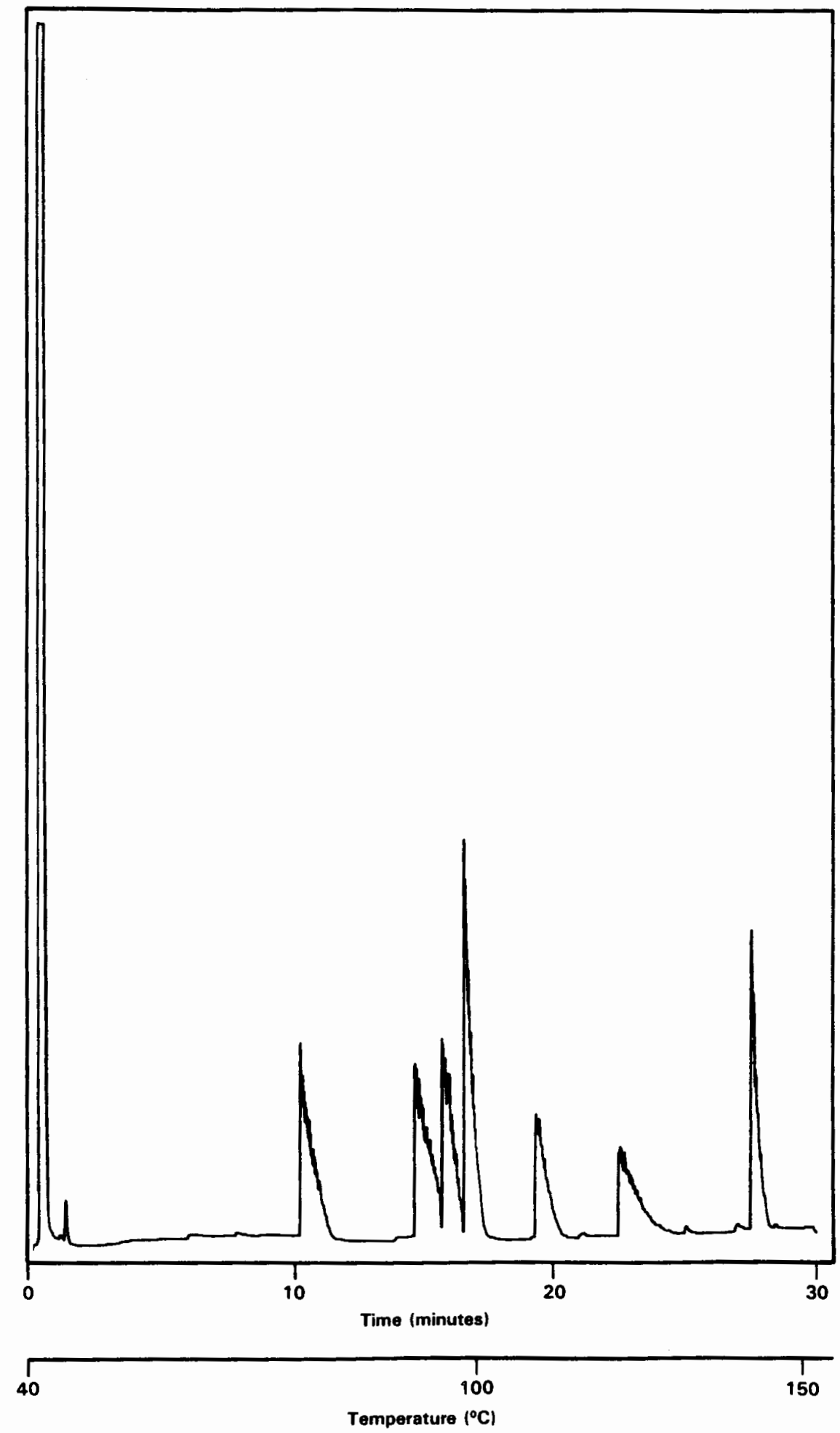

FIGURE 13. Capillary Gas Chromatography Test Chromatogram of a "Polarity Test" Mixture. Conditions and peak identifications are the same as in Figure 12. 


\section{Maximizing Crosslinking Efficiency}

Investigations conducted to determine conditions required for maximizing the crosslinking efficiency (both internal crosslinking of the stationary phase and crosslinking to the deactivation layer) have included: optimizing the treatment conditions for chemically generated free-radical initiators; the use of gamma-irradiation to induce crosslinking (Hubbal et al., 1984); and the use of both techniques combined. A series of studies were undertaken to systematically define the treatment characteristics that were critical for achieving high-quality column performance. The important parameters were the surface water and impurity concentration on the silica surface; the purity, concentration, and age of the methylene chloride solution of polyhydrosiloxane polymers; the removal of the solvent from the thin polyhydrosiloxane film produced during dymanic coating; the temperature and duration of the polyhydrosiloxane reaction; and the purity of the azo-t-butane crosslinking reagent. Conditions were also defined that allowed the stationary-phase polymers to be loaded with the maximum amount of crosslinking agent. (Too much will obstruct and eventualiy plug the small-bore column.) Thermal treatment conditions were also optimized to generate a maximum flux of free radicals.

Gamma-irradiation from a ${ }^{60} \mathrm{Co}$ source at 5- and 10-Mrem doses were applied over several-hour periods in another set of experiments designed to determine if the gamma-irradiation induced crosslinking greater than that obtained with the chemical free-radical initiators. Investigations (e.g., rotating the column around its center axis) aimed at improving the uniformity of the irradiation dose were also conducted. Improvement in total crosslinking, as evidenced by increased stationary-phase stability, has been observed for the chemically crosslinked and gamma-irradiated columns.

\section{FABRICATION OF FLUID FLOW RESTRICTORS}

One important detail in high-performance SFC is the need to maintain a constant pressure on the fluid throughout the length of the analytical column in order to eliminate fluctuations in fluid density. Thus, the pressure drop that occurs across the column should be confined to the immediate end of the column across a very short distance compared to the length of the column. 
Ideally, a point-source flow restrictor would allow all the pressure drop to occur at a single point at the end of the column. However, the diameter of the point-source flow restrictor hole required for a 25- to 50- $\mu \mathrm{m}$-ID column is less than $1 \mu \mathrm{m}$, a diameter very prone to plugging and difficult to manufacture reproducibly.

Because the flow restriction was a crucial aspect of attaining good SFC performance, one research area of this program has been to investigate the design and fabrication of flow restrictors for use at the end of SFC columns. The technique of providing flow restriction previously used was to use a small ZDV butt connector to attach a length of 5- $\mu \mathrm{m}$-ID fused silica tubing to the

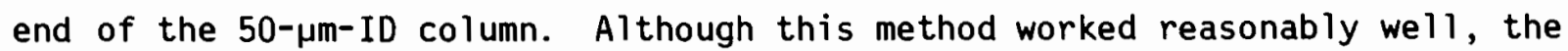
need for the butt connector placed limitations on the interface designs. Our first approach toward manufacturing a restrictor that did not require a butt

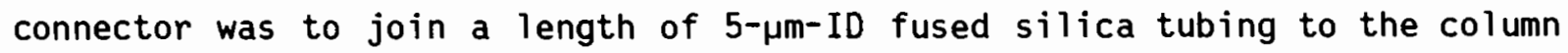
tubing, using a 250- $\mathrm{mm}-\mathrm{ID}$, fused silica sleeve, into which both smaller tubes fit nicely. Polyfurfuryl alcohol was used as the sealant because of its high chemical resistance and high temperature utility. We found that we could create unions which were reasonably serviceable, although the 250- $\mu \mathrm{m}$-ID sleeve limited the pressure capability to less than the 200 atm desired for use with supercritical fluids.

Probably the most logical way to provide a restriction is to heat the fused silica tube and draw it to a smaller diameter. If the restriction were drawn at the end of the column, no butt connector would be required. Flames tried as heat sources were uncontrollable for heating such small fused silica tubes. A tiny electric furnace was built, which provided a controlled, known, high-temperature environment. In operation, a 50- $\mu \mathrm{m}-\mathrm{ID}$, fused silica tube was placed in the furnace, and a weight was attached to one end, which caused the tubing to stretch and draw to a smaller diameter. Operating this furnace at $1300^{\circ} \mathrm{C}$ produced slowly tapering restrictors that appeared to be usable. The restrictors withstood $110 \mathrm{~atm}$ with no apparent damage and produced restricted flows at this pressure equivalent to those produced by a $20-\mathrm{cm}$ length of $5-\mu \mathrm{m}-$ ID tubing. Faster-tapering restrictors were produced by operating the furnace at $1500^{\circ} \mathrm{C}$; these were pressure-tested to 200 atm with no apparent problems. However, these restrictors, even though closer in design to an ideal point- 
source restrictor compared to the longer-taper type, did not provide adequate flow restriction for the present instrumental designs.

Since restrictor flow characteristics suitable for SFC/MS had been achieved with lengths of 5- to 8-cm narrow-bore capillary tubing, we anticipated that a more practical, shorter restrictor might be a $1-\mathrm{cm}$ length of the smallest reproducibly attainable internal diameter (approximately 2 to $3 \mu \mathrm{m}$ ). This short length of restrictor proved to be difficult to reproduce, and our most recent efforts have concentrated on producing slowly tapering nozzles, 2 to $3 \mathrm{~cm}$ long. These nozzles are manufactured quite reproducibly using a $3-\mathrm{g}$ weight and operating the furnace at $1275^{\circ} \mathrm{C}$. Flow testing of capillary restrictors, which had been successfully used in SFC and SFC/MS experiments, indicated that a restrictor that permitted a nitrogen gas flow of 0.1 to 1 $\mathrm{cm}^{3} / \mathrm{min}$ at a high-side pressure of $800 \mathrm{psi}$ was desired. The length of the 3-cm nozzles can easily be trimmed to produce restrictors with flow characteristics that fall in the useful range. Restrictors of this type are more robust than the fast-taper variety made previously, and therefore are more easily handled without breakage. The furnace is now used routinely to manufacture this type of flow restrictor for a variety of SFC and SFC/MS equipment. 


\section{$\underline{\text { REFERENCES }}$}

Fjeldsted, J. C., W. P. Jackson, P. A. Peaden, and M. L. Lee. 1983. J. Chromatogr. Sci. 21: 222-225.

Hubba1, J. A., P. R. DiMauro, E. F. Barry, E. A. Lyons, and W. A. George. 1984. J. Chromatogr. Sci. 22: 185-191.

Kong, R. C., C. L. Woolley, S. M. Fields, and M. L. Lee. 1984. Chromatographia 18: $362-366$.

McLafferty, F. W. 1983. Tandem Mass Spectrometry. John Wiley \& Sons, Inc., New York, NY.

Smith, R. D. and H. R. Udseth. 1983a. Anal. Chem. 55: 2266-2272.

Smith, R. D. and H. R. Udseth. 1983b. Sep. Sci. Technol. 18: 245-252.

Stark, T. J., R. D. Dandeneau, and L. Mering. 1980. In: Proceedings, 1980 Pittsburgh Conference, p. 2, March 10, 1980, Atlantic City, NJ. ABC Press, Monroeville, PA.

Woolley, C. L., R. C. Kong, B. E. Richter, and M. L. Lee. 1984. JHRC\&CC 7 : 329-332.

Wright, B. W., P. A. Peadon, M. L. Lee, and G. M. Booth. 1982a. Chromatographia 15: 584-586.

Wright, B. W., P. A. Peadon, M. L. Lee, and T. J. Stark. 1982b. J. Chromatogr. 248: 17-34. 
PNL-5214

UC-90

\section{DISTRIBUTION}

\author{
OFFSITE \\ James Carr \\ U.S. Department of Energy \\ Fossil Energy \\ MS-FE-42 \\ Washington, DC 20545 \\ Chief, Distribution Branch \\ U.S. Department of Energy \\ Office of Scientific and Technical \\ Information \\ P. 0. Box 62 \\ Oak Ridge, TN 37831 \\ Stanley J. Dapkunas \\ U.S. Department of Energy \\ Fossil Energy \\ MS-C- 155 \\ Washington, DC 20545 \\ DOE Technical Information Center (30) \\ Dave Dubis, Project Manager \\ U.S. Department of Energy \\ Morgantown Energy Technology Center \\ P. 0. Box 880 \\ Morgantown, WV 26505 \\ Thomas W. Keech, Jr., Chief \\ Instrumentation Branch \\ U.S. Department of Energy \\ Morgantown Energy Technical Center \\ P. 0. Box 880 \\ Morgantown, WV 26505
}

Arthur J. Liberatore, Chief

Components Section

U.S. Department of Energy

Morgantown Energy Technical Center

P. 0. Box 880

Morgantown, WV 26505

Jan J. Wachter, Chief

Analytical Sciences Branch

U.S. Department of Energy

Morgantown Energy Technical Center

P. 0. Box 880

Morgantown, WV 26505

ONSITE

DOE Richland Operations office

J. J. Sutey

A. DeGrazia

Pacific Northwest Laboratory

L. R. Bunnell

E. K. Chess (10)

R. D. Smith

B. W. Wright

Biology Publications Office

Technical Information (5)

Publishing Coordination (2) 
,

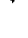

